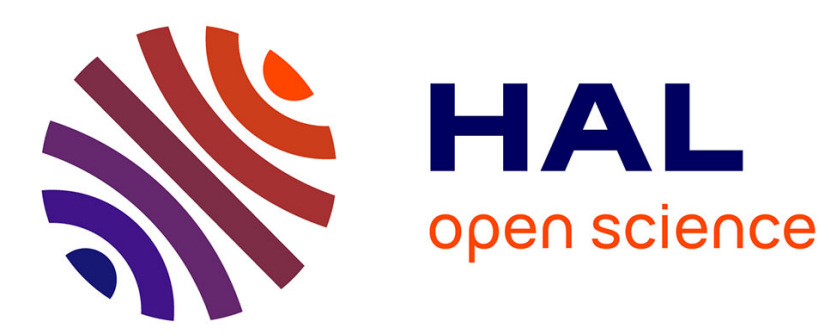

\title{
What can we learn from the exclusion volume of fluctuations and precipitates?
}

Joel Lepinoux

\section{To cite this version:}

Joel Lepinoux. What can we learn from the exclusion volume of fluctuations and precipitates?. Philosophical Magazine, 2021, 101 (9), pp.1098-1118. 10.1080/14786435.2021.1882710 . hal-03254885

\section{HAL Id: hal-03254885 \\ https://hal.science/hal-03254885}

Submitted on 9 Jun 2021

HAL is a multi-disciplinary open access archive for the deposit and dissemination of scientific research documents, whether they are published or not. The documents may come from teaching and research institutions in France or abroad, or from public or private research centers.
L'archive ouverte pluridisciplinaire HAL, est destinée au dépôt et à la diffusion de documents scientifiques de niveau recherche, publiés ou non, émanant des établissements d'enseignement et de recherche français ou étrangers, des laboratoires publics ou privés. 


\title{
What can we learn from the exclusion volume of fluctuations and precipitates?
}

\author{
J. Lépinoux
}

\section{ARTICLE HISTORY}

Compiled 10th December 2020

Univ. Grenoble Alpes, CNRS, Grenoble INP, SIMaP, F-38000 Grenoble, France

Corresponding author: joel.lepinoux@grenoble-inp.fr

\begin{abstract}
In the literature, the volume of a cluster (i.e. a fluctuation of the solid solution or a precipitate) is identified as the volume of a sample of the bulk precipitate phase containing the same number of solute atoms (i.e. of the same size). This approximation is suitable for macroscopic precipitates but is debatable for a rigorous description of microscopic clusters, especially during the nucleation and growth stages. To go beyond this simplification, this work focuses on the notion of 'exclusion volume', a volume which accounts for real cluster configurations in a given 3D lattice. The exclusion volume normalized by the cluster size is a complex function of the cluster size, which, like the cluster free energy, evolves during precipitation kinetics and converges toward an asymptote in the long-time limit. Furthermore, this asymptote exhibits a bifurcation which clearly separates fluctuations from precipitates, for a cluster size which is found equal to the minimum value of the critical size for nucleation. With help of Atomistic Kinetic Monte Carlo (AKMC) simulations it is shown that the evolution of cluster volume during precipitation can be accurately predicted.
\end{abstract}

Keywords: AlLi alloys, precipitation, computer simulation, clusters, Monte Carlo

\section{Introduction}

This work intends to contribute to the necessary improvement of the phenomenological laws used to describe the different stages of precipitation in classical numerical approaches as the so-called KWN framework [1, 2]. These methods are powerful and versatile but various ingredients of the required constitutive laws implicitly assume a high dilution or/and a low supersaturation. Thus, to build more robust laws, the study of concentrated binary alloys is a privileged means, likely to raise information which was out of reach until recently.

The major improvements in Statistical Physics methods about 40 years ago [3, 4], then in simulation techniques at the atomic scale [5] and more recently in calculation capabilities have allowed the emergence of such studies. Most recent advances in this direction are derived from data provided by Atomistic Kinetic Monte Carlo (AKMC) simulations, e.g. [6], and the formalism of Cluster Dynamics (CD) $[7,8]$ is used as a bridge between the atomistic world and the phenomenological approaches.

Whatever the formalism, the system is implicitly assumed at equilibrium, which in practice means quasi-equilibrium (QE); cf. [9] for details about this notion. This means that the initial state introduced in $\mathrm{CD}$ or KWN frameworks should be taken beyond the onset of QE: when AKMC simulations can be applied, this is the most basic result one can expect from such simulations. But AKMC simulations can also provide the three key ingredients which are necessary to make CD able to reproduce the AKMC cluster distributions during precipitation kinetics, with a high accuracy:

(i) the free energy of clusters (the main ingredient), as a function of cluster size, temperature and solute concentration [10]

(ii) the volume of clusters, used for various purposes (the present work)

(iii) the concentration of pure matrix sites, a multiplicative factor ignored in classical models and close to 1 in very dilute alloys (work in progress). 
Therefore, the practical goal of the present work is to describe the volume of clusters during precipitation. If a cluster is defined as a set of connected atoms (the details of the rules depend on the crystallographic structure - cf. next section), the description of fluctuations and precipitates in precipitation models depends on the underlying formalism. For instance, in CD [7] there is only one type of objects, i.e. clusters, whose properties depend only on their size (i.e. the number of solute atoms they contain). In the KWN framework [1, 2], the main objects are precipitates, whether they are growing or dissolving and the solid solution is considered as a reservoir of solute atoms, disregarding the details of its distribution. Each approach has advantages and drawbacks but both treat the cluster volume as a minor ingredient (cf. Section 2.4).

Basic data required to run AKMC simulations are recalled in section 2.1. The chemical potential and the critical size for nucleation playing an important role in this work, these notions are recalled in sections 2.2 and 2.3 respectively, in relation with AKMC simulations. Then, before presenting results about the notion of exclusion volume introduced in section 2.4, we first consider the cluster enthalpy, a quantity related to, but independent of the exclusion volume.

\section{Methods and definitions}

\subsection{AKMC simulations}

To obtain generic results, this type of study requires a model material as simple as possible. In particular:

the size misfit between the two types of atom has to be negligible, at least in the considered range of temperature

- the solubility limit has to be high

- monomers should be the only class of mobile clusters.

Of course it would be easy to consider 'numerical alloys' specially built for this purpose but a few real alloys are acceptable candidates. Apart its industrial interest, the main advantage of considering a real alloy is the possibility to compare with experimental and theoretical works even if the main goal is not to obtain a perfect agreement.

For these reasons ordered and coherent $\mathrm{L}_{2} \delta^{\prime} \mathrm{Al}_{3} \mathrm{Li}$ clusters in aluminium have been chosen as a model case to study precipitation in concentrated binary alloys, using AKMC simulations (for details about this technique see e.g. [11]). For this purpose, an atomic model accounting for first- and secondnearest-neighbours interactions (denoted hereafter NN1 and NN2, respectively) has been built (for details see in particular Tables 1 and 2 in [12]). It is based on the parametrization proposed by Garland and Sanchez [13] for the two effective pair interaction parameters, i.e. $\omega_{1}=-3742 \mathrm{~J} / \mathrm{mol}$ for NN1 and $\omega_{2}=1871 \mathrm{~J} / \mathrm{mol}$ for NN2. As required, this atomic model reproduces various thermodynamic and kinetic properties of this alloy. Diffusion is described through vacancy exchanges with one of its twelve NN1, using the residence time algorithm like in dilute alloys [14], and there is no attempt to account for the local configuration around the saddle point. It was carefully checked through diffusion experiments that monomers are the only mobile clusters. There is little doubt that this alloy is much more complex than what this basic atomic model can describe, but provided it reproduces some fundamental properties [12], it is sufficient for our purpose.

AKMC simulations have been performed on a rigid lattice (for an introduction about this technique see e.g. [11]), using boxes of either $200^{3}$ atoms or $500^{3}$ atoms, depending on conditions and purposes. Typical snapshots are shown in section 3.3. As in a recent work dedicated to cluster free energy [10], we consider 2 cases at $T=85^{\circ} \mathrm{C}$ and 2 cases at $T=200^{\circ} \mathrm{C}$ (the solubility limits are respectively $2.7 \%$ and $6.3 \%$ - all concentrations mentioned in this paper are atomic concentrations):

(1) $T=85^{\circ} \mathrm{C}$ and $C_{0}=4.75 \%$, moderate concentration and moderate supersaturation $(+2.05 \%)$

(2) $T=200^{\circ} \mathrm{C}$ and $C_{0}=8.25 \%$, high concentration and moderate supersaturation $(+1.95 \%)$

(3) $T=200^{\circ} \mathrm{C}$ and $C_{0}=10 \%$, high concentration and high supersaturation $(+3.7 \%)$

(4) $T=85^{\circ} \mathrm{C}$ and $C_{0}=9 \%$, high concentration and very high supersaturation $(+6.3 \%)$

The first and second cases exhibit a classical behaviour during precipitation, i.e the well-known stages of nucleation, growth and coarsening are easily identified. On the contrary, for the two other cases it is no longer possible to distinguish these different stages, like in the situations described by Robson [15]. 


\subsection{The chemical potential}

To highlight these two typical behaviours one possible way consists in reporting the evolution of the chemical potential of monomers as a function of time. Following the notations introduced in [10], we write:

$$
\bar{\mu}_{1}=\bar{h}_{1}+\ln \left(\frac{C_{1}}{M_{1}}\right)
$$

where $\bar{x}$ denotes the quantity $x$ normalised by $k_{B} T\left(k_{B}\right.$ is the Boltzmann constant and $T$ is the temperature); $h_{1}$ is the monomer enthalpy, $C_{1}$ is the monomer concentration and $M_{1}$ is the concentration of pure matrix sites [9]. Replacing a pure matrix site by a solute atom results in a monomer and vice versa. Then, with $\bar{\mu}_{1}=\bar{\mu}_{1}^{S L}$ at the solubility limit (SL), $\Delta \mu=\bar{\mu}_{1}-\bar{\mu}_{1}^{S L}$ is the excess of chemical potential, i.e. the excess of the term $\ln \left(C_{1} / M_{1}\right)$.

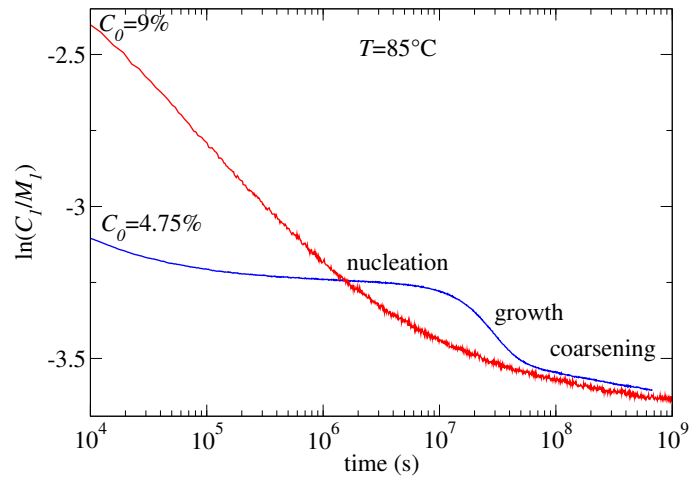

(a)

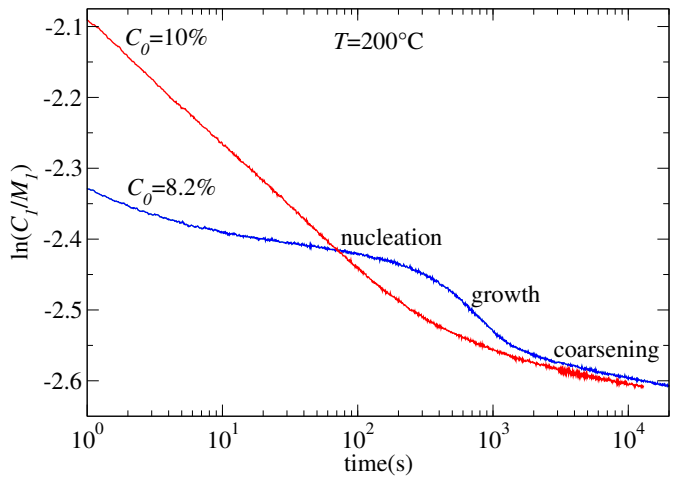

(b)

Figure 1.: The reduced chemical potential of monomers $\ln \left(C_{1} / M_{1}\right)$ vs. time for 2 concentrations and 2 temperatures: (a) $T=85^{\circ} \mathrm{C}$, (b) $T=200^{\circ} \mathrm{C}$. The two low supersaturated cases exhibit 3 well-defined stages (blue) as in dilute alloys while this distinction vanishes for high supersaturated cases (red).

\subsection{The critical size for nucleation}

Although the notion of critical size $n^{*}$ or critical radius $r^{*}$ for nucleation is not directly related to the goal of the present work, its usefulness will appear in section 3. In classical models using the capillary approximation to describe the cluster free energy, the critical radius is readily obtained [7]. In these models, the critical size evolves during precipitation according to the underlying model chosen to describe the driving force for precipitation (e.g. a regular solution) and to the value imposed for the free interface energy, assumed constant.

This procedure is simple but implicitly assumes that the critical size is very large, which supposes a very low supersaturation. In CD, the evolution of the cluster distribution is controlled by the so-called emission and absorption coefficients, i.e. $\alpha_{n}$ and $\beta_{n}$, respectively [7]. It has been shown that these coefficients are related to quantities which can be directly extracted from AKMC simulations [10, 12]:

$$
\left(\frac{\beta_{n}}{\alpha_{n+1}}\right)=\left(\frac{C_{1}}{M_{1}}\right)\left(\frac{P_{n \rightarrow n+1}^{*}}{P_{n+1 \rightarrow n}^{*}}\right)
$$

where $P_{n \rightarrow n+1}^{*}$ and $P_{n+1 \rightarrow n}^{*}$ are respectively the capture and release coefficients, related to the cluster free energy $F_{n}^{*}$ : 


$$
\left(\frac{P_{n \rightarrow n+1}^{*}}{P_{n+1 \rightarrow n}^{*}}\right)=\exp \left(\frac{h_{1}}{k_{B} T}\right) \exp \left(-\frac{F_{n+1}^{*}-F_{n}^{*}}{k_{B} T}\right)
$$

Note the symbol * which indicates that the 'CapRel' method used to calculate $P_{n \rightarrow n+1}^{*}$ and $P_{n+1 \rightarrow n}^{*}$ accounts for the probability of coagulation with other clusters around when the considered cluster captures a new solute atom (see [12] for details about this method).

Strictly speaking, the critical size $n^{*}$ is the value of $n$ which satisfies $\alpha_{n}=\beta_{n}$ [7]. Knowing that $\beta_{n}$ is proportional to the cluster radius $R_{n}$, with Equations (2-3), the condition $\alpha_{n}=\beta_{n}$ writes:

$$
\left(\frac{P_{n-1 \rightarrow n}^{*}}{P_{n \rightarrow n-1}^{*}}\right)=\left(\frac{M_{1}}{C_{1}}\right)\left(\frac{R_{n-1}}{R_{n}}\right)
$$

The ratio $\left(R_{n} / R_{n-1}\right)$ is of course very close to 1 , excepted for small clusters. To safely use this equation to get $n^{*}$, the cluster distribution must be continuous at least up to a few ten classes beyond $n^{*}$ because Equation (4) refers to two contiguous classes of cluster size, $n$ and $n-1$. And ideally, the last classes in this range should contain at least a few clusters. These two conditions are always satisfied at the beginning of the four cases investigated here, thus Equation (4) is suitable for our present needs. It will be shown elsewhere that for larger clusters it is necessary to adopt other strategies to derive $n^{*}$, depending on its magnitude.

Whatever the imposed conditions (i.e. concentration and temperature), all AKMC simulations reported here have been launched from a random state (pseudo infinite temperature). In these conditions, $n^{*}$ always starts from a virtually infinite value. Then, when imposing a finite and constant temperature, $n^{*}$ decreases very quickly while the system starts to organize. The most important point for our present concern is that $n^{*}$ reaches a minimum, which will be denoted $n_{0}^{*}$. Then, what happens after is very dependent on conditions and will be detailed elsewhere. Another point worth mentioning for the understanding of the next section is that the lower the supersaturation, the lower the fraction of super-critical clusters when $n^{*}$ reaches its minimum $n_{0}^{*}$, i.e. it tends to vanish like in the classical case of very dilute alloys. And of course, on the contrary, the higher the supersaturation, the higher the fraction of super-critical clusters when $n^{*}=n_{0}^{*}$.

\subsection{The exclusion volume}

If $C_{p}$ is the solute concentration in the bulk precipitate phase at the solubility limit, the total number of sites per solute atom is $N_{p}=1 / C_{p} ; N_{p}=4.135$ at $85^{\circ} \mathrm{C}$ and $N_{p}=4.415$ at $200^{\circ} \mathrm{C}$. In classical models the total number of sites associated with a n-mer is $n N_{p}$ at any time. This defines a volume $V(n)=n N_{p} \Omega$ where $\Omega$ is the atomic volume ( $\Omega=l^{3} / 4$ for the FCC lattice where $l$ is the lattice parameter). In other words, no matter their size or whether they are fluctuations of the solid solution, growing or dissolving precipitates: all clusters are considered as samples of the bulk precipitate phase.

The notion of 'exclusion' has brought a different point of view about cluster volume [16]. The 'exclusion volume' of a cluster is composed of its solute skeleton and of all matrix NN1 and matrix NN2 of these atoms. These sites are involved when a solute atom leaves or is captured by a cluster or in some changes of configuration at constant size. In practice, we prefer to use the number of excluded sites $N_{e x}(n)$ instead of the associated volume $V_{e x}(n)$ (which can be easily derived from $N_{e x}(n)$ assuming spherical clusters). For instance, for a monomer in the $\mathrm{Al}$ matrix, $N_{e x}(1)=19$ : the monomer itself, its 6 matrix NN2 and its 12 matrix NN1. Apart from the monomer, $N_{e x}(n)$ is a function of temperature and at a lesser extent of the concentration.

To describe the evolution of $N_{e x}(n)$ it is more convenient to consider its normalized form $X(n)=$ $N_{e x}(n) / n$. Indeed, we already know the limit of this quantity for large cluster size in the long-time limit: it is the same value than at the solubility limit [12]. Note that in a discrete world, this definition means that any cluster is surrounded by a shell free of solute atoms, whose thickness is about the lattice parameter, as can be seen in Figure 2.

As a consequence for concentrated alloys, the sum of exclusion volumes might exceeds the total volume of the system. Indeed, at high concentrations, most exclusion sites at the cluster-matrix interface 


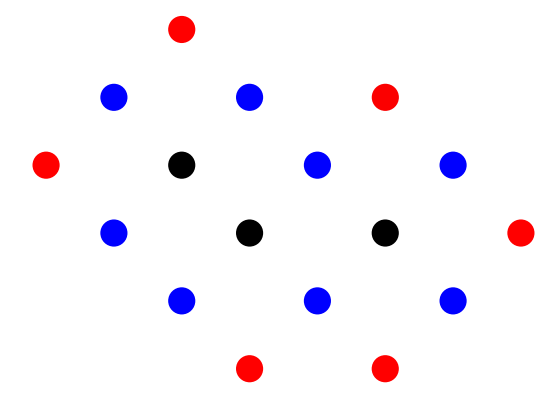

Figure 2.: An example of 3-mer configuration in 2D. Black disks: the solute skeleton of the cluster, defining a solute NN1 pair and a solute NN2 pair. Blue disks: sites which have at least one NN1 bond with a solute atom. Red disks: other sites which have at least one NN2 bond with a solute atom. All these sites contribute to the exclusion volume of the cluster. For this $2 \mathrm{D}$ configuration, $N_{e x}(3)=17$.

are likely to be shared among several clusters but should be accounted for only once. This is the difficulty of modelling $M_{1}$ which will be discussed elsewhere. Thus, the effective cluster radius $R_{n}$ which enters in the expression of $\beta_{n}[7]$ or used to calculate the precipitate volume fraction, for instance, is not the radius of the exclusion volume $R_{e x}(n)$ but derives from this one as follows:

$$
R_{n}=R_{e x}(n)-\lambda
$$

Where $\lambda$ is taken constant whatever the value of $n$ and the radius of the exclusion volume writes:

$$
R_{e x}(n)=l\left(3 N_{e x}(n) / 16 \pi\right)^{1 / 3}
$$

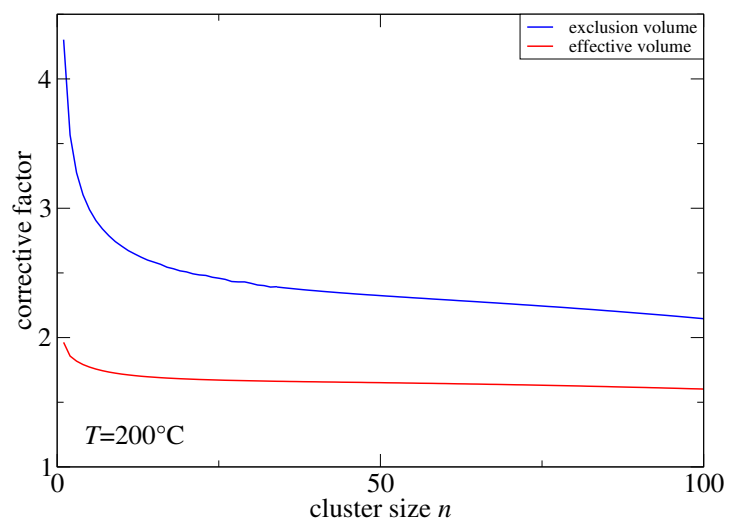

Figure 3.: Comparison of the exclusion volume and the effective volume, normalized by $\left(n N_{p} / 4\right) l^{3}$,i.e. the volume attached to $n$ solute atoms in the bulk precipitate phase. The effective volume is calculated with $\lambda=0.24 l$ which corresponds to 8.666 sites for a monomer while $N_{e x}(1)=19$ and $N_{p}=4.415$ at $200^{\circ} \mathrm{C}$.

Various arguments can be invoked to set up the value of $\lambda$, which can be seen as a way to adjust the thickness of the interface between a cluster and the matrix while the classical description of cluster volume simply neglects the interface. Based on a naive geometrical criterion, it was first taken equal to $0.52 l$ in [16] but a recent modelling of $M_{1}$ based on AKMC data leads to $0.24 l$ (work in progress). It is important to note that whatever the criterion adopted to set up the value of $\lambda$ (which is not important for our present purpose), the exclusion volume remains the fundamental quantity from which the effective radius $R_{n}$ is derived. Figure 3 compares the exclusion volume and the effective volume, both 
normalized by the volume associated with the same number of solute atoms in the bulk precipitate phase. As can be seen, the corrective factor for the effective volume is small and converges toward 1 in the large-cluster limit, but very slowly. Thus, this correction concerns mostly nucleation and growth as expected and becomes negligible for macroscopic precipitates.

These volumes can hardly be compared with experimental measurements, whether they are based on MET [17] or more recently on APT [18]. Whatever the technique, the obtained information is not yet as reliable as the one immediately available in atomistic simulations. When such a comparison becomes meaningful, experiments and simulations data will have to be analysed for various cluster sizes, using exactly the same tools to avoid artefacts of methodology.

\section{Results}

\subsection{Cluster enthalpy}

To put into evidence the physical nature of the exclusion volume, we first consider a quantity which can be unambiguously defined: the cluster enthalpy, a function directly related to the number of solute - matrix pairs $\left(N_{N N 1}\right.$ and $\left.N_{N N 2}\right)$ and to the two effective pair parameters $\omega_{1}$ and $\omega_{2}$ :

$$
H(n)=\omega_{1} N_{N N 1}+\omega_{2} N_{N N 2}
$$

In fact $H(n)$ itself is rather confusing while its two components reveal interesting features. Thus the contributions of first-near-neighbours $\left(N_{N N 1}\right)$ and second-near-neighbours $\left(N_{N N 2}\right)$ of the solute skeleton of a cluster as functions of the cluster size $n$ are presented separately in Figure 4; each symbol represents the average value for a class of cluster size $n$.

Figure 4(a,c,e), related to NN1 bonds, describe mostly bulk properties. Note that $N_{N N 1}$ is always close to 12 (exactly 12 for a monomer, by definition), the asymptotic limit in the $\mathrm{L}_{2}$ structure. The deviation from this value measures the probability to find two solute atoms of a same cluster linked by a NN1 bond. This situation being energetically unfavourable, its occurence is a function of temperature, cluster size and time. For instance the range of variation is 3 times larger at $200^{\circ} \mathrm{C}$ than at $85^{\circ} \mathrm{C}$ and the long-time convergence towards the asymptote occurs for larger clusters. Starting from the monomer, this probability increases up to a maximum around $n=5-10$ (except for the first curve in Figure 4e), then it decreases. The apparently odd behaviour of the first curve in Figure 4e is only a consequence of the initial state, which is purely disorderd, and that this curve corresponds to an extended distribution and a short time. This NN1 disorder is mostly a surface feature, which explains the role of cluster size and also temperature. The evolution with time means that rearrangements occur even inside clusters and that progressively this disorder vanishes. Thanks to an elementary experiment on a bi-phased system (precipitate / matrix) at equilibrum, we have calculated that the vacancy spent about $2 \%$ of its time within the $\mathrm{L}_{2}$ phase, or equivalently, the concentration of vacancies inside precipitates is about $2 \%$ of its value in the matrix. With time, it is sufficient to reach the equilibrium density of the bulk precipitate phase, but this is a slow process.

Figure 4(b,d,f), related to NN2, describes mostly the cluster-matrix interface. By definition the monomer has 6 matrix NN2 bonds and the large-size assymptote is now close to 0 (NB: 0 corresponds to equilibrium of the bulk precipitate phase at $0 \mathrm{~K}$ ). Note a bifurcation near $n_{0}^{*}$. Using equations recalled in section 2.3 and simulations data, we found that this value $n_{0}^{*}$ is simply the minimum of $n^{*}$. Thus, in first approximation, the left parts of Figures 4 are related to fluctuations of the solid solution while the right parts are related to precipitates. In details it depends on the imposed conditions. In case (1), when $n^{*}$ reaches $n_{0}^{*}$, the average size of the few super-critical clusters is very close to $n_{0}^{*}$. By consequent, almost all precipitates are formed from clusters smaller than $n_{0}^{*}$, following the classical scenario. In case (2), when $n^{*}$ reaches $n_{0}^{*}$, the number of supercritical clusters is already noticeable. Note that in Figure $4 d$ the bifurcation seems to occur for a value of $n$ slightly larger than $n_{0}^{*}$. Finally, in case (3) there is no classical nucleation at all, most precipitates are already supercritical when $n^{*}$ reaches $n_{0}^{*}$. This is associated with a more important translation of the bifurcation beyond $n_{0}^{*}$. This translation is not observed in case (4) at $85^{\circ} \mathrm{C}$, although the supersaturation is much higher. 
It is worthy to note that in the long-time limit all $N_{N N 2}$ values tend to align along a curve, whether precipitates are growing or dissolving, although the dispersion is larger than for fluctuations.

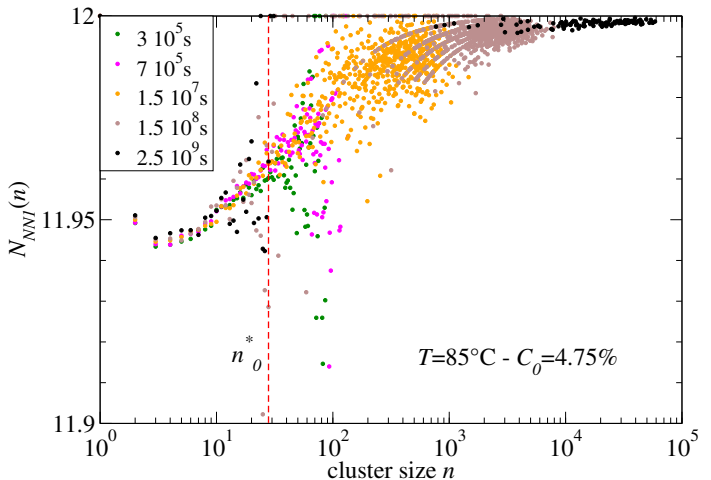

(a)

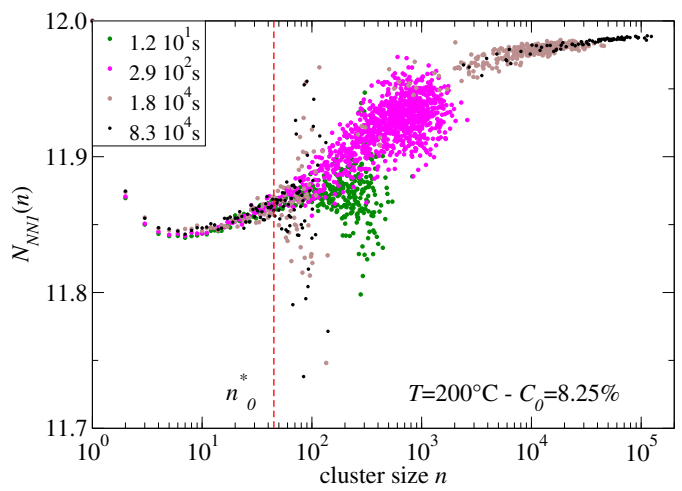

(c)

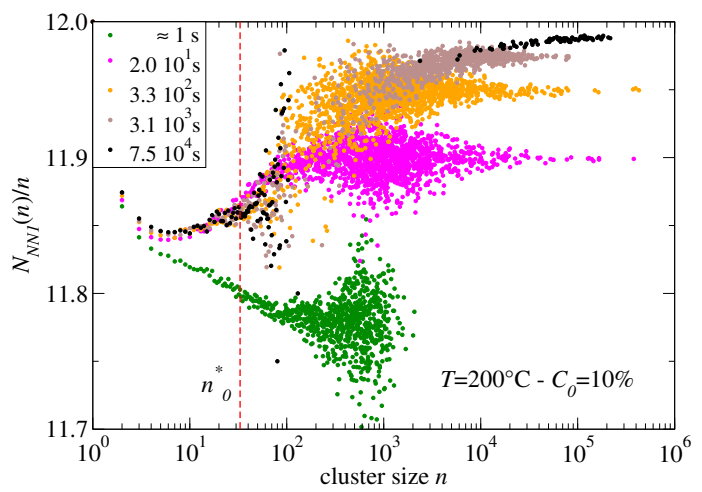

(e)

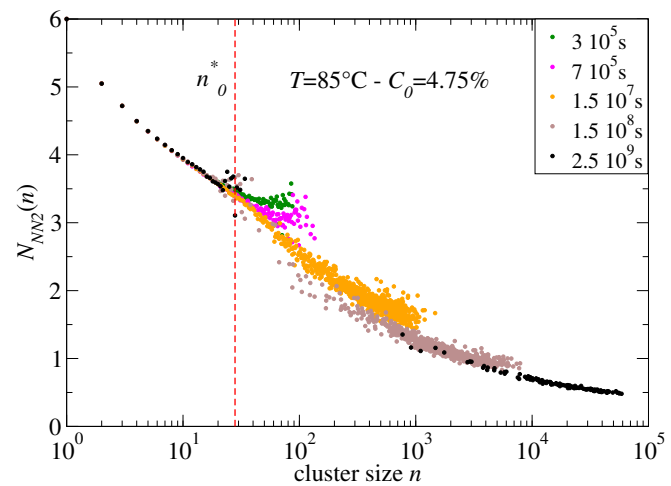

(b)

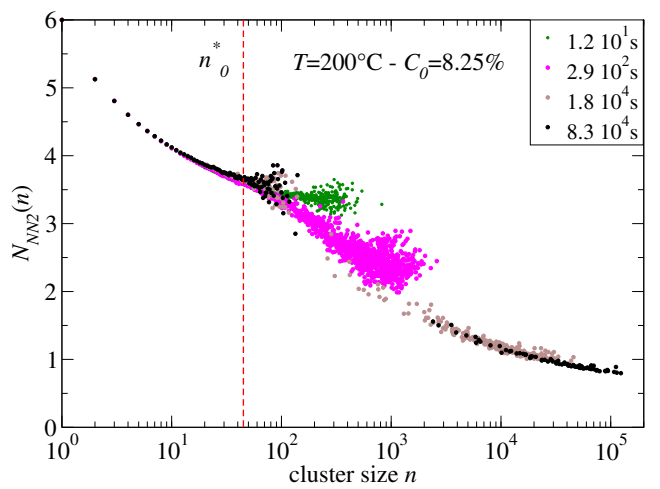

(d)

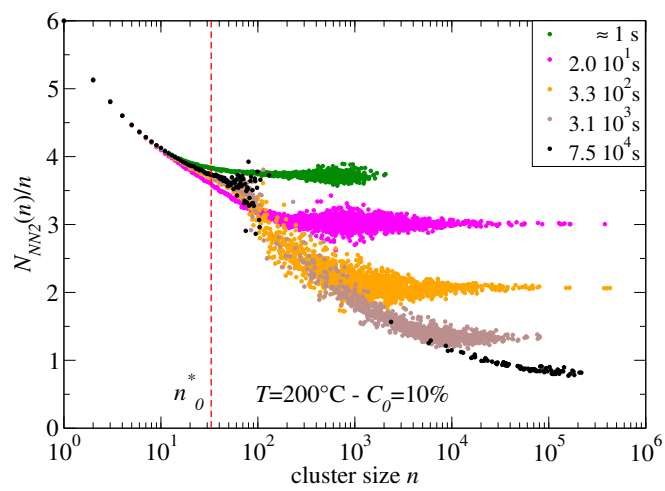

(f)

Figure 4.: Average number of NN1 bonds (a,c,e), NN2 bonds (b,d,f) between solute atoms of clusters of size $n$ and the matrix, at different times (cf. Figure 1). $n_{0}^{*}$ is the minimum of the critical size at the very beginning of nucleation. Simulation box size: $[500]^{3}$. (a,c,e) and (b,d,f) refer to cases (1-3) respectively.

\subsection{The exclusion volume at moderate supersaturation}

In this section we focus on the two first cases defined in section 3.1. Comparing Figure 5(a-b) and Figure4(a-d) comfims that the exclusion volume is based on physical grounds. For instance, $X(n)$ 
versus $n$ exhibits a clear change of behaviour for the same value of $n$ than the enthalpy, i.e. near $n_{0}^{*}$. Figure 5 suggests that, in first approximation, $X(n)$ could be approximated by the blue curve for $n<n_{0}^{*}$ (fluctuations) an by the red one for $n>n_{0}^{*}$ (precipitates).

These two curves are built as follows:

- the 'fluctuations' curve corresponds to the exclusion volume of clusters at the solubility limit, supposed to be known [12]. Then, if $n_{0}^{*}$ is also known, the value of $X\left(n_{0}^{*}\right)$ is straightforward.

- the 'precipitates' curve starts at $\left(n_{0}^{*}, X\left(n_{0}^{*}\right)\right)$ and converges toward the value of $X(n)$ in the bulk precipitate phase (a quantity which is also supposed to be known like other properties at equilibrium) when $n \rightarrow \infty$.

In the next figures these two asymptotic curves are built according to these principles, which are further detailed in section 3.4.

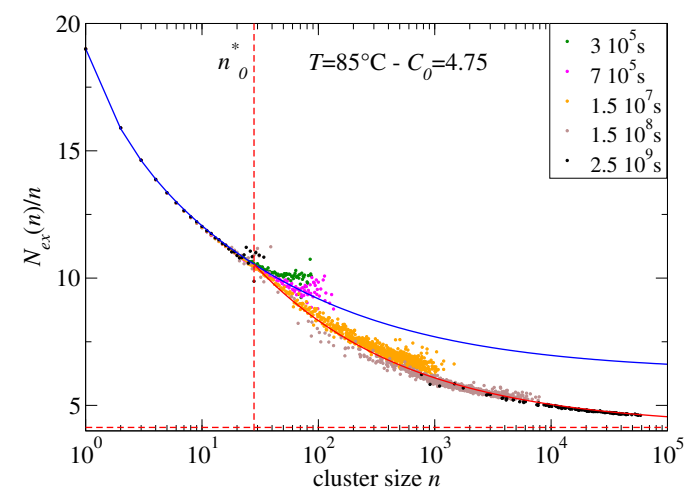

(a)

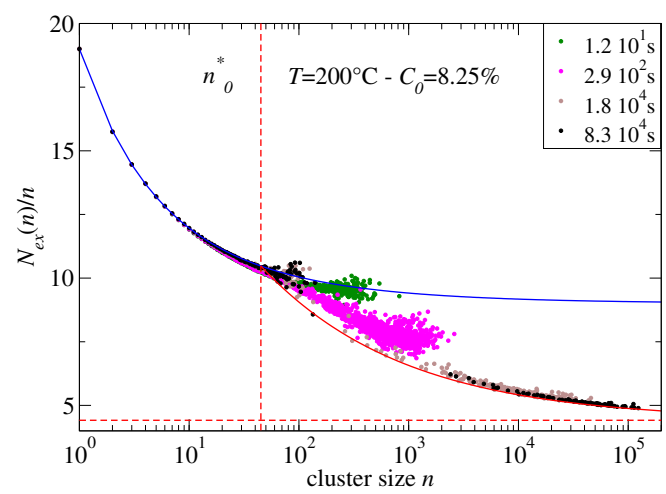

(b)

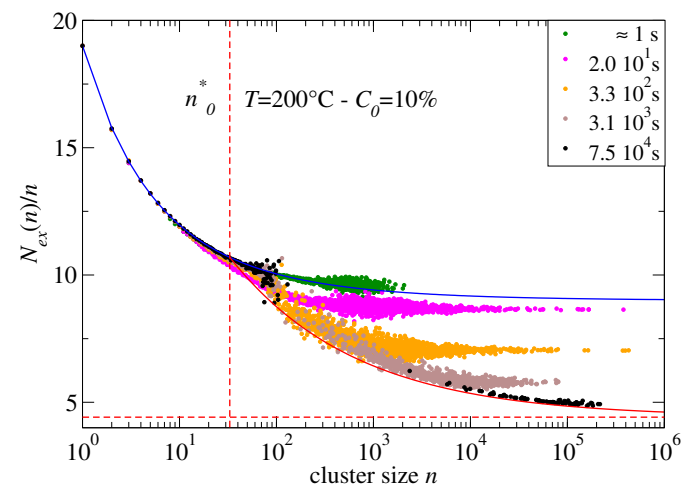

(c)

Figure 5.: Evolution of the normalised number of excluded sites vs. cluster size, associated to the evolution of the numbers of NN1 and NN2 bonds reported in figure 4(a-f). The blue curves are related to fluctuations of the solid solution at the solubility limit, the red curves are the asymptotes for precipitates and the horizontal dashed lines correspond to the bulk precipitate phases. $n_{0}^{*}$ is the minimum of the critical size $n^{*}$ at the very beginning of nucleation ( $1^{\text {st }}$ curve). Simulation box size: $[500]^{3}$. (a-c) refer to cases (1-3) respectively.

For the last curves in Figure 5a it can be noted that dissolving precipitates, mostly in the range of size [30 - 300] are more compact than predicted by the asymptote (red curve). The emission probability of solute atoms at the interface is a function of their binding energy with the cluster. Therefore, clusters emit in priority the atoms of lower binding energy, which tends to increase the solute concentration at the interface of dissolving clusters, especially at low temperature. However, this small deviation related to dissolving precipitates can be ignored for modelling purposes.

As expected, due to a comparable supersaturation, case (2) is very similar to the case (1). Of course, 
fluctuations are much more extended at $200^{\circ} \mathrm{C}$ than at $85^{\circ} \mathrm{C}$, which increases the difference between the two asymptotic curves for $n>n_{0}^{*}$. As mentioned in the previous section, note that when $n^{*}=n_{0}^{*}$, the number of super-critical clusters is now significant; the distribution extends up to a few $n_{0}^{*}$ as can be seen in Figure 5b. Coagulation is more noticeable than in case (1) but it is still a secondary effect which can be ignored.

Although these alloys are too much concentrated to be considered as a dilute alloys, their behaviour follows the classical scenario: most precipitates form when the size of clusters reaches the critical size. The deviation observed around the queue of the curve means that, at the beginning of nucleation, super-critical fluctuations are not as dense as would be fluctuations of the same size at the solubility limit. Note that the volume of clusters of size $n^{*}<n_{0}^{*}$ is already close to that of fluctuations of same size at the solubility limit (the blue curve). Then, with increasing time, precipitates become more compact than would be fluctuations of same size, and in the growth stage, $X(n)$ converges toward the precipitate asymptote (the red curve). This trend continuously increases during coarsening and of course the last curves concern also a good number of dissolving precipitates.

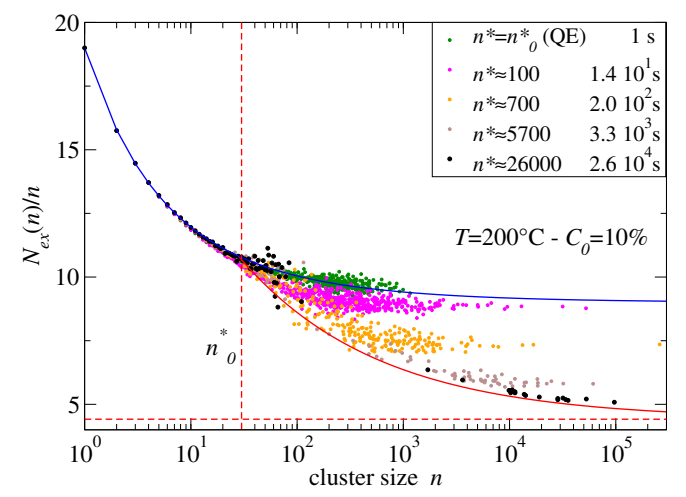

(a)

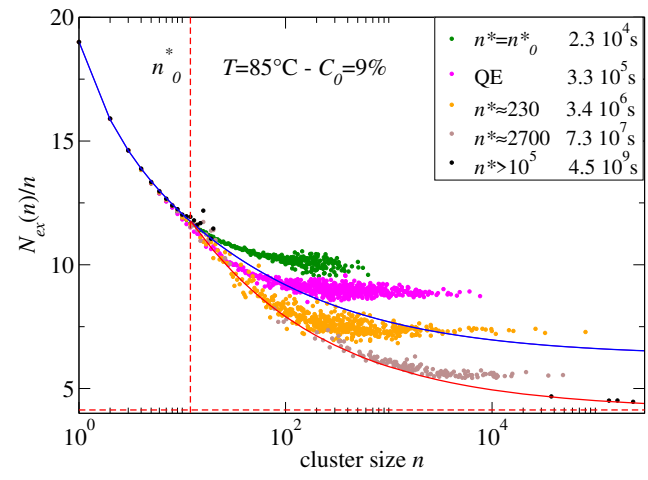

(b)

Figure 6.: Evolution of the normalised number of excluded sites vs. cluster size, during a precipitation kinetics. (a) can be compared with figure 5(c) for very close times but larger simulation box. The blue curves are related to fluctuations at the solubility limit, the red curve are the asymptotes for precipitates and the horizontal dashed line correspond to the bulk precipitate phases. $n_{0}^{*}$ is the minimum of the critical size $n^{*}$ at the very beginning of nucleation ( $1^{\text {st }}$ curve). Simulation box size: $[200]^{3}$.

\subsection{The exclusion volume at high supersaturation}

One of the most important characteristics of cases (3) and (4) is that almost all precipitates are already formed and are growing when $n^{*}$ reaches its minimum value. This explains the absence of a large plateau in Figure 1 corresponding to the nucleation stage in cases (1) and (2). As can be noted in Figure 6(a-b), the queue of the distributions becomes horizontal, a behaviour which can be also observed in Figure 4(e-f).

As the amount of information increases with the size of the simulation box, this trends is more obvious in Figure 5(c) ([500] $]^{3}$ atoms) than in Figure 6(a) ([200] $]^{3}$ atoms). The comparison of these two figures shows that this behaviour is not an artefact due to a too small simulation box in Figure 6(a-b). This means that clusters whose size is of the order of $n^{*}$ (which corresponds roughly to the beginning of the plateau) connect each other to form larger groups of clusters, wich possibly connect each others and so on. The brigdes between clusters are too tiny to modify the density of the resulting groups of clusters, which explains the horizontal extension of these plateaus. Note that their lengths increase up to a maximum, before decreasing. 


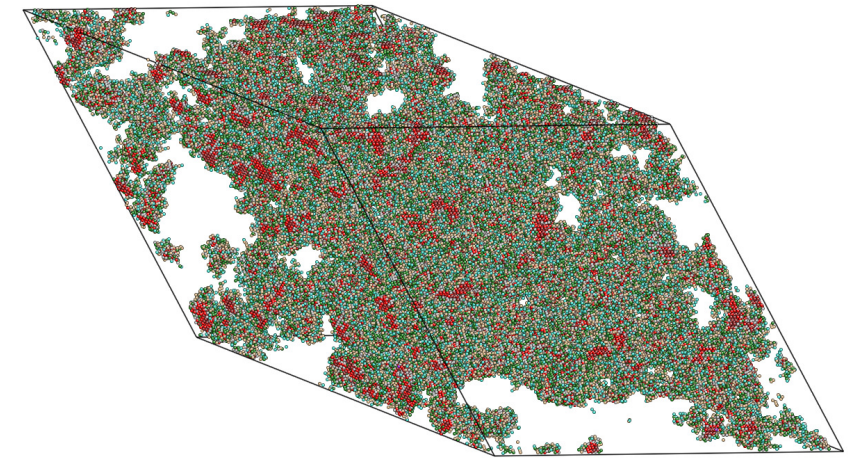

(a)

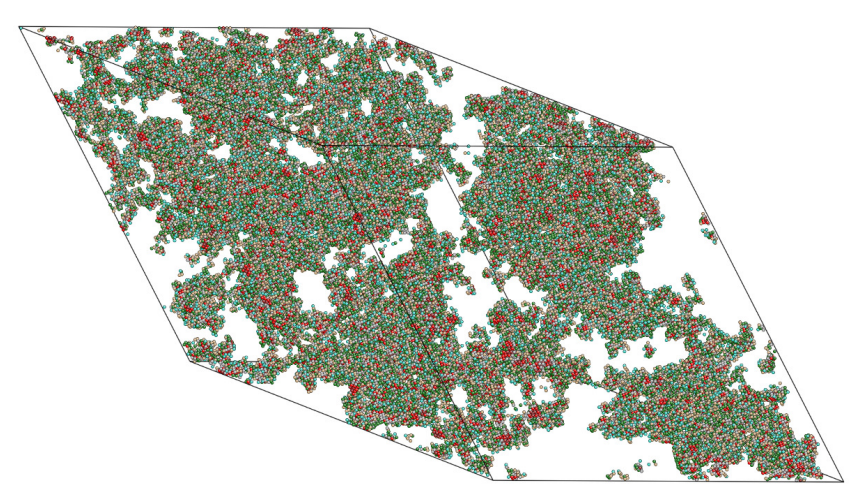

(c)

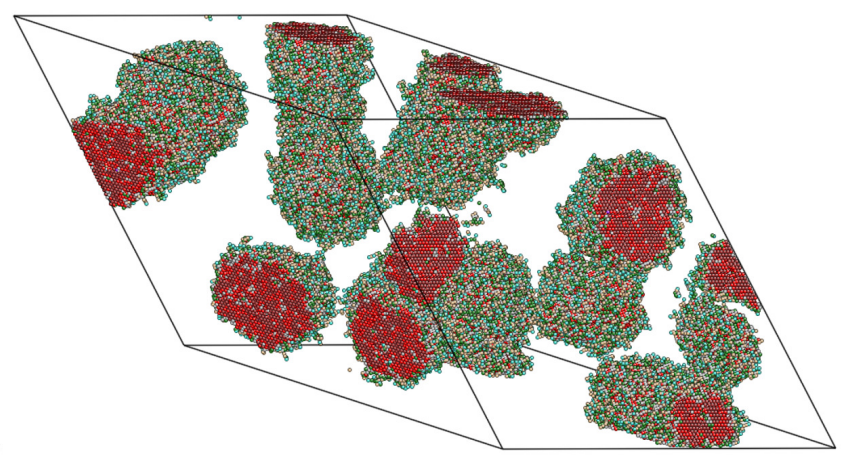

(b)

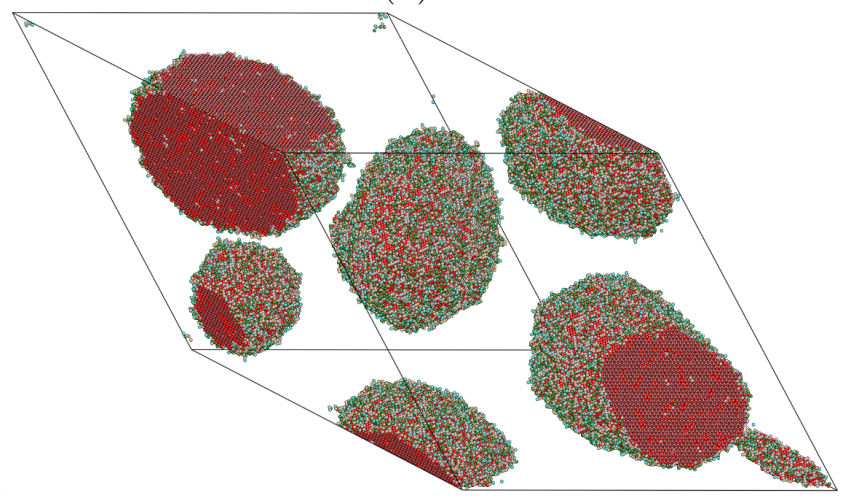

(d)

Figure 7.: Snapshots taken from simulation boxes of [200] $]^{3}$ atoms using Atomeye [22] (atom colours are related to their number of NN2 (k denotes 1000):

(a-b) $T=200^{\circ} \mathrm{C}, C_{0}=10 \%$.

(a) $\log \left(C_{1} / M_{1}\right)=-2.38$; largest super-cluster: $260 \mathrm{k}$ solute atoms.

(b) $\log \left(C_{1} / M_{1}\right)=-2.361$; largest clusters from $9 \mathrm{k}$ to $90 \mathrm{k}$ solute atoms (cf. last curve in Figure 6a) (c-d) $\mathrm{T}=85^{\circ} \mathrm{C}, \mathrm{C} 0=9 \%$.

(c) $\log \left(C_{1} / M_{1}\right)=-3.18$; largest super-cluster: $130 \mathrm{k}$ solute atoms.

(d) $\log \left(C_{1} / M_{1}\right)=-3.66$; only 4 precipitates left: $28 \mathrm{k}, 133 \mathrm{k}, 165 \mathrm{k}$ and $242 \mathrm{k}$ solute atoms (cf. last curve in Figure 6b).

This behaviour is the signature of a strong occurrence of coagulation between close clusters, which leads to the formation of colonies of connected clusters and even a super-colony spreading over the whole system as can be seen in Figures $7 \mathrm{a}$ and $7 \mathrm{c}$. These snapshots have been taken when the largest cluster colony has its maximum size with a simulation box of $[200]^{3}$ atoms. At $200^{\circ} \mathrm{C}$ this cluster colony contains about $1 / 3$ of solute atoms which represents about $90 \%$ of the supersaturation. At $85^{\circ} \mathrm{C}$, the maximal density of the largest colony is $50 \%$ smaller, it contains about $11 \%$ of solute atoms which represents $16 \%$ of the supersaturation. These cluster colonies appear during the time range corresponding to the nucleation stage at $4.75 \%$ (cf. Figure 1) then they fragment, first in smaller groups of clusters untill they condense into ordinary clusters near the beginning of the coarsening stage (see Figures $7 \mathrm{~b}$ and 7d). Again, this trend to form super-clusters is not an artefact resulting from the small size of the simulation box, nevertheless it does exist an artefact: the formation of a super-cluster spreading over the whole system. This results from both the finite size of the simulation box and the periodic boundary conditions. The comparison of results obtained with the two sizes of simulation box in case (4) has revealed that the size of the largest cluster is not proportional to the size the simulation box. Instead of a widespread cluster colony, with a $[500]^{3}$ atoms box, one observes many clusters whose size is of the order of a few $10^{5}$ atoms. At $200^{\circ} \mathrm{C}$ these large clusters have time to connect each others before the triggering of fragmentation, but not at $85^{\circ} \mathrm{C}$, unless the simulation box is small enough. Nevertheless, this topological artefact does not have any consequence on the precipitation kinetics, all comparable curves superimpose.

A few decades ago, a work based on an average field approach of precipitation [19] triggered a rich controversy about the nature of transformations occurring in these concentrated ordered alloys 
(i.e. ordering vs. clustering). A study based on AKMC simulations concluded that ordering and phase separation occur concurrently and that usual scaling laws apply to the late stages of the precipitation kinetics [20], in agreement with TEM observations [21]. Although we did not explicitly explore ordering from a quantitative point of view, the complete cluster distributions observed in this work are in agreement with this first AKMC study.

However it should be recalled that in the present work, by convention, a solute atom can be connected to a cluster either by a NN1 bond or by a NN2 bond. Consequently, a cluster can be composed of several connected sub-clusters which do not necessarily lie on the same sub-lattice and the bridge between two sub-clusters can be reduced to only one solute atom. Therefore, this definition is likely to promote a 'coagulation stage', as observed. Note that coagulation has already been observed by TEM [17], thus it is not an artefact of AKMC simulations.

Accounting for coagulation and fragmentation in CD is possible but very cumbersome (e.g. see [7]) and probably not appropriate to model such cluster colonies. Again, CD is the best tool to test various ways to cope with such unusual situations and decide if it is justified, and efficient, to introduce coagulation and fragmentation processes. Otherwise there is a much simpler solution: running AKMC simulations until most colonies of clusters break down. Of course this solution has a cost in terms of computing time. Then most of the coarsening stage can be modelled by CD or even with the KWN formalism, more efficient in the long-time limit, provided it can be launched from a correct cluster distribution and that cluster properties can be properly set up.

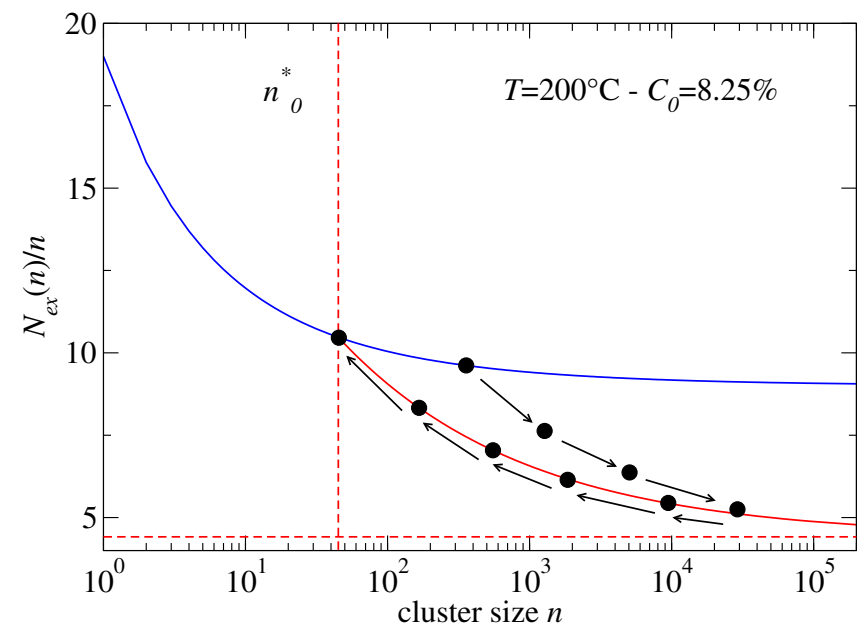

Figure 8.: Evolution of the normalized number of excluded sites of a fictitious cluster during the precipitation kinetics. Starting from the fluctuations asymptote, it decreases while the size increases and converges toward the precipitate asymptote. Here, we assume that this cluster becomes subcritical when its size reaches about $310^{4}$ atoms. During dissolution, $X(n)$ increases, following the precipitate asymptote until, again, it cannot be distinguished from fluctuations. Same conventions than in Figure 5 and 6

As can be seen in figures related to cases $(2-3)$ at $200^{\circ} \mathrm{C}, n_{0}^{*}$ is not a strict limit for the solid solution during the whole kinetics, neither $X\left(n_{0}^{*}\right)$ is constant if one looks into details, while at $85^{\circ} \mathrm{C}$ this approximation is convenient even in case (4). Two reasons can explain this difference of behaviour. When the system precipitates, more space becomes available (e.g. the factor $\mathrm{M}_{1}$ continuously increases), then fluctuations can expand and explore configurations of higher energy and larger volume (see [9] for details). Supercritical clusters which are already available when the critial size reaches its minimum value require much less reorganization before starting to grow at $200^{\circ} \mathrm{C}$ than at $85^{\circ} \mathrm{C}$. An alternative criterion to $n_{0}^{*}$ for the bifurcation observed in figures $4-5$ at $200^{\circ} \mathrm{C}$ is the average size of clusters larger than $n_{0}^{*}$ when $n^{*}=n_{0}^{*}$. This criterion gives 65 and 98 instead of 45 and 33 for cases (2) and (3) respectively. This is in better agreement with observations at $200^{\circ} \mathrm{C}$ than the $n_{0}^{*}$ criterion but the improvement does not justify the use a sophisticated criterion which depends on temperature. 
Compared with the over-simplified treatment of cluster volume in classical modelling of precipitation, we beleive that trying to account for all these features would be counterproductive. Nevertheless, it is interesting to understand that these small deviations are not artefacts of AKMC simulations and that they could be included if it appears necessary in future.

\subsection{Predicting the evolution of $X(n)$ during precipitation kinetics}

The challenge consists now in predicting the evolution of these exclusion volumes, knowing the final states (from preliminary studies of equilibrium states at the solubility limit, cf. [12]) and the beginning of the kinetics from AKMC simulations (at least up to the onset of QE). Indeed, the goal of modelling is to save as much computing time as possible while providing results as close as possible to what would give AKMC simulations.

First we need to explain how to define the two asymptotic curves reported in Figures 5-6. Then it will be shown how to build predictable fits of $X(n, t)$.

\subsubsection{Fluctuations}

Like the free energy, the exclusion volume of clusters at the solubility limit is well described by a polynomial function [16], which leads for $X(n)$ :

$$
X(n)=a+b n^{-1 / 3}+c n^{-2 / 3}+d \log (n) / n
$$

where $a$ is a volume term equal to $N_{p}$, while fitting AKMC data provides $b, c$ and $d$, related to surface, edges and corners respectively. For modelling purposes one can assume that the volume of fluctuations can be described by the same law at any time; compared to other sources of error, this approximation has negligible effects. In other words, in first approximation, fluctuation volumes do not depend on solute concentration.

\subsubsection{Precipitates}

To describe $X_{\infty}(n)$, the asymptotic curve for precipitates, we could use Equation (8) again, of course with another set of coefficients, but we have checked that the last two terms can be neglected. The advantage of this simplification is that the curve becomes easy to build as it requires only two conditions: $X_{\infty}(n)=X_{\infty}\left(n_{0}^{*}\right)$ when $n=n_{0}^{*}$ and $X_{\infty}(n)=N_{p}$ in the large-cluster size limit. Thus, the asymptotic behaviour of precipitates can be described as follows:

$$
X_{\infty}(n)=N_{p}+\left(X\left(n_{0}^{*}\right)-N_{p}\right)\left(n_{0}^{*} / n\right)^{1 / 3}
$$

As can be seen in Figures 5-6 this expression provides good fits for very large precipitates as well as for dissolving ones. $N_{p}$ is supposed to be known, then one only needs to get $n_{0}^{*}$ from AKMC simulations each time the imposed conditions change, using Equation (4). Equation (9) can be rewritten in a more convenient form for further comparisons:

$$
X_{\infty}(n)=X\left(n_{0}^{*}\right)+\left(X\left(n_{0}^{*}\right)-N_{p}\right)\left(\left(n_{0}^{*} / n\right)^{1 / 3}-1\right)
$$

As can be seen in Figures 5-6, the function $X(n)$ is not constant during the kinetics. Figure 8 illustrates this effect for a fictitious cluster: the difficulty is the description of the first part of its history, i.e. between the two asymptotic curves.

To get good fits, starting from a function like Equation (8), we have found necessary and sufficient to use the first three terms where $a$ is set equal to $X\left(n_{0}^{*}\right)$. But compared with Equations (9) one more parameter means that one more condition is required to predict the fits. We could not find any simple additional condition but we have remarked that $c \approx\left(b_{\infty}-b\right)$ where $b_{\infty}$ is the value of $b$ in the long-time limit. Thus, this relation has been imposed to eliminate one parameter and we have checked 
a posteriori that this simplification does not lower the quality of fits. Finally, the fit of $X(n)$ can be written:

$$
X(n)=X_{\infty}(n)+\varepsilon\left(\left(n_{0}^{*-1 / 3}-n^{-1 / 3}\right)-\left(n_{0}^{*-2 / 3}-n^{-2 / 3}\right)\right)
$$

where $\varepsilon$ is a positive parameter to be derived from the fits.

The results are presented in Figure 9 as a function of $\log \left(C_{1} / M_{1}\right)$ (cf. Equation(1)). By construction, all curves end at $\varepsilon=0$ (on the left) for the value taken by $\log \left(C_{1} / M_{1}\right)$ at the solubility limit (i.e. -3.69 at $85^{\circ} \mathrm{C}$ and -2.65 at $\left.200^{\circ} \mathrm{C}\right)$. Due to the risk of bias, measurements of $X(n)$ taken before the system reaches $\mathrm{QE}$ should be disregarded (except in one case, as shown in Appendix).

Owing to the special behaviour of cases (3) and (4), trying to fit the complete $X(n)$ curves, i.e. including the long final plateau at intermediate times, seems a meaningless strategy. Nevertheless it is possible and interesting to compare the beginning of these curves with cases (1) and (2), as reported in Figure 9 (i.e. from $n_{0}^{*}$ up to the onset of the plateau, which corresponds roughly to $n^{*}$ as can be seen in Figure 8).

The next step is the prediction of $\epsilon$ while minimizing the amount of necessary information, contrary to the fits shown in Figure 9 which have been built using all known data. As indicated in introduction, one task of AKMC simulations is to provide initial cluster distributions taken at least up to the onset of QE. Such a configuration is also mandatory to correctly predict the evolution of cluster free energy during precipitation [10]. For these two tasks, the larger the size of the simulation box, the better the results. But of course, as the computing time is proportional to this size, we have to find a compromise between the computation time and the accuracy of results: for this purpose we have chosen a box size of $500^{3}$ atoms. But if we don't have any information beyond the onset of QE, the transition between the linear and the non-linear parts of the curves presented in Figure 9 has to be guessed. Of course, if one knows the whole curves, a posteriori, this seems fairly easy with a limited risk of error (cf. Appendix). Therefore, the knowledge of one case can benefit other similar cases with a minimum of information.

But there is a less risky strategy. Nowadays, even personal computers offer the possibility to run several calculations simultaneously. Thus, the proposed method is the following: for each simulation performed up to the onset of QE with a $500^{3}$ atoms box, another simulation is performed on another node, in the same conditions, but with a smaller box size. And both simulations can be stopped together. Again there is a compromise to find about the size of this second box between the quality of outputs (which is in favour of large boxes) and the proportion of the precipitation kinetics which can be explored (which is in favour of small boxes): for this purpose we have adopted a box size of $200^{3}$ atoms.

In average, when large box simulations are stopped around the onset of QE, small box simulations are already around the transition between nucleation and growth, which is sufficient to prevent bad choices to predict $\epsilon$ (cf. Appendix). It will be shown in a next paper that this strategy is also suitable to get the third ingredient required to run $\mathrm{CD}$, i.e. $M_{1}$ (cf. Introduction).

\section{Discussion}

We have shown that considering the volume of clusters as a physical property instead of a simple geometrical property reveals interesting features and major differences between fluctuations and precipitates. The key quantity to consider is the exclusion volume, whose behaviour is very similar to that of the NN2 component of cluster enthalpy. The effective volume of clusters, used for instance to define the precipitated fraction, can be derived from the exclusion volume, but not directly from the cluster size. More generally, the ability of AKMC to provide well-defined data allows for various kinetic aspects of precipitation to be revisited. And for this purpose, the use of a physically based definition of cluster volume is one of the essential elements to reach an accurate agreement between AKMC and models.

The normalized exclusion volume $X(n)$ vs. the cluster size exhibits a discontinuity which is can be identified to $n_{0}^{*}$, the minimum value of the critical size: only clusters of size smaller than $n_{0}^{*}$ never experiment pure growth. This defines a two-branches asymptotic curve for $X(n)$, i.e. one for for fluctuations and another one for precipitates. 


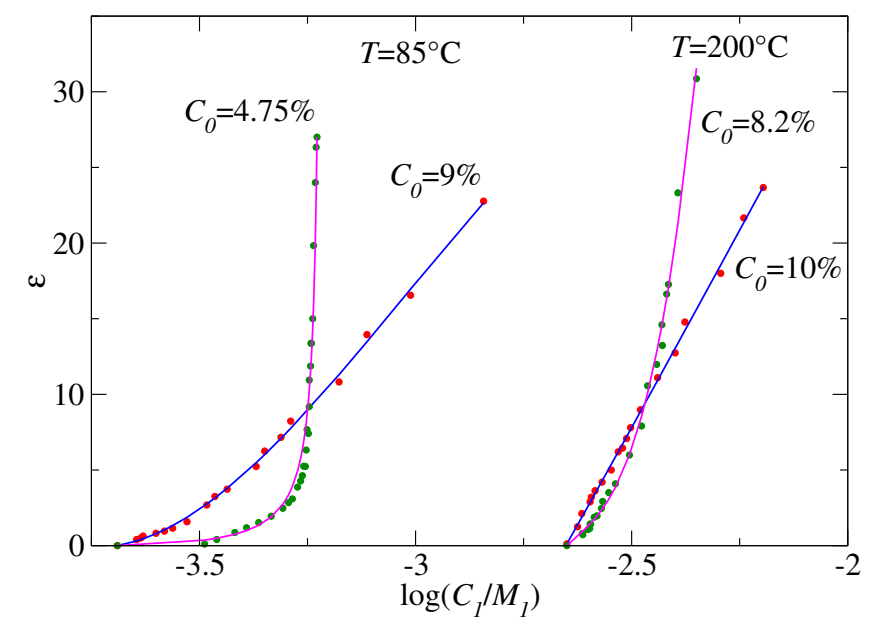

Figure 9.: Evolution of $\varepsilon$ versus $\log \left(C_{1} / M_{1}\right)$ during precipitation. Symbols: measured data from AKMC simulations. Curves start around the onset of QE (right tips) and ends for the value of $\log \left(C_{1} / M_{1}\right)$ at the solubility limit $\left(-3.69\right.$ for $T=85^{\circ} \mathrm{C}$ and -2.65 for $\left.T=200^{\circ} \mathrm{C}\right)$.

In first approximation, $X(n)$ can be considered constant for fluctuations during the whole precipitation kinetics, taking the same values as at the solubility limit. In details it is of course more complex but this approximation should be convenient for most modelling needs.

Provided the supersaturation is not too high, modelling the evolution of the precipitate branch of $X(n)$ during the precipitation kinetics is fairly simple. As for the cluster free energy [10], the results can be expressed as functions of the excess of chemical potential $\Delta \mu$.

At high supersaturation, the extensive coagulation of precipitates leads to the formation of large cluster colonies which progressively condense in ordinary clusters near the beginning of the coarsening stage. Nevertheless, even in such complex situations, it is still possible to describe $X(n)$ up to $n^{*}$, and beyond this value $X(n)=X\left(n^{*}\right)$ is an excellent approximation.

During dissolution, in first approximation $X(n)$ follows the asymptotic curve in the reverse direction, up to the bifurcation between fluctuations and precipitates. Thus, as soon as the size of clusters reaches $n_{0}^{*}$, dissolving precipitates should be considered again as fluctuations, instead of managing their dissolution until they reduce to monomers, as usually done.

Although these results have been obtained considering a particular ordered alloy, the main features are believed to be general. Even the treatment of precipitation in dilute alloys could take advantage of these results after some simplification (i.e. the case (1) is a good starting point). There is no particular difficulty to account for these new features into classical models to improve their agreement with AKMC simulations.

When $M_{1}$, the third and last ingredient (cf. Introduction) required to run CD is available, the challenge will be to find the best way to cope with coagulation and fragmentation of cluster colonies at high supersaturation.

\section{Acknowledgements}

Dr. E. Clouet is gratefully acknowledged for providing his AKMC package. Dr C. Sigli is warmly acknowledged for fruitful discussions and suggestions about this paper.

\section{References}

[1] R. Wagner and R. Kampmann, Homogeneous second phase precipitation, in Materials Science and Technology, Eds. R. W. Cahn, P. Haasen, and E. J. Kramer, Vol. .5, Chapt. 4, Verlag Chemie GmbH, Weinheim (1991) pp. 213 - 304.

[2] M. Perez, M. Dumont and D. Acevedo, Implementation of classical nucleation and growth theories for precipitation, Acta Materialia 56 (2008) pp. 2119-2132 
[3] D.P. Landau and K. Binder, A guide to Monte Carlo simulations in statistical physics, Cambridge University Press (2014).

[4] C. Bennett, Efficient estimation of free energy differences from Monte Carlo data, Journal of Computational Physics 22 (1976) pp. 245-268.

[5] D. Frenkel and B. Smit, Understanding molecular simulation - From algorithms to applications, Academic Press, San Diego, CA (2001).

[6] T. Jourdan, J.-L. Bocquet and F. Soisson, Modelling homogeneous precipitation with an event-based Monte Carlo method: Application to the case of Fe-Cu, Acta Materialia 58 (2010) pp. 3295-3302.

[7] E. Clouet, Modeling of nucleation processes, in Fundamentals of Modelling for Metals Processing, D.U. Furrer and S.L. Semiatin (Eds.), Materials Park, OH, ASM Handbook, vol. 22A Fundamentals of modelling for metals processing (2010) pp.203-219.

[8] E. Clouet, A. Barbu, L. Laë and G. Martin, Precipitation kinetics of $\mathrm{Al}_{3} \mathrm{Zr}$ and $\mathrm{Al}_{3} \mathrm{Sc}$ in aluminium alloys modeled with cluster dynamics, Acta Materialia 53 (2005) pp. 2313-2325.

[9] J. Lépinoux and C. Sigli, Multiscale modelling of precipitation in concentrated alloys: from atomistic Monte Carlo simulations to cluster dynamics: I thermodynamics, Phil. Mag. 98(1) (2017) pp. 1-19.

[10] J. Lépinoux, Evolution of clusters free energy during precipitation in concentrated binary alloys, Phil. Mag. Letters, (2020) DOI: 10.1080/09500839.2020.1777340.

[11] F. Soisson and G. Martin, Monte Carlo simulations of the decomposition of metastable solid solutions: Transient and steady-state nucleation kinetics, Phys. Rev. B 62 (2000) pp. 203-214.

[12] J. Lépinoux and C. Sigli, Extracting free energy of clusters in concentrated binary alloys from atomistic Monte Carlo simulations, Modelling. Simul. Mater. Sci. Eng. 27 (2019) pp. 085001-(1-22)

[13] J.S. Garland, J.M. Sanchez, Cluster variation method calculation of the metastable aluminiumlithium phase diagram, in Kinetics of Ordering Transformations in Metals, H. Chenand V.K. Vasudevan (Eds.), TMS, Warrendale, PA (1992) pp. 207-216.

[14] A. Clouet, M. Nastar and C. Sigli, Nucleation of $\mathrm{Al}_{3} \mathrm{Zr}$ and $\mathrm{Al}_{3} \mathrm{Sc}$ in aluminium: from kinetic Monte Carlo simulations to classical theory, Phys Rev. B 69 (2004) pp. 064109-(1-14)

[15] J.D Robson, Modelling the overlap of nucleation, growth and coarsening during precipitation, Acta Materialia 52 (2004) pp. 4669-4676.

[16] J. Lépinoux, Contribution of matrix frustration to the free energy of cluster distributions in binary alloys, Phil. Mag. 86 (2006) pp. 5053-5082.

[17] K. Mahalingam, B.P. Gu, G.L. Liedl and T.H. Sanders Jr, Coarsening of $\delta^{\prime}\left(\mathrm{Al}_{3}\right.$ Li $)$ precipitates in binary Al-Li alloys, Acta metallurgica 35(2) (1987) pp.483-498.

[18] W. Lefebvre, F. Danoix, G. Da Costa, F. De Geuser, H. Hallem, A. Deschamps and M. Dumont, 3DAP measurements of Al content in different types of precipitates in aluminium alloys, Surf Interface Anal 39 (2007) pp. 206-212.

[19] A.G. Khachaturyan, T. E Lindsey and J. W. Morriss Jr., Theoritical inverstigations of the precipitation of $\delta$ ' in Al-Li, Metallurgical Transactions A (1988) pp. 249-258

[20] T.A. Abinandanan, F. Haider and G. Martin, Computer simulations of diffusional phase transformations: Monte Carlo algorithm and application to precipitation of ordered phases, Acta Mater. 12 (1998) pp. 4243-4255.

[21] G. Schmitz, K. Hono and P. Haasen, High resolution electron microscopy of the early decomposition stage of Al-Li alloys, Acta metall. Mater. 42 (1994) pp. 201-211.

[22] J. Li, AtomEye: an efficient atomistic configuration viewer, Modelling. Simul. Mater. Sci. Eng. 11 (2003) pp. 173-177.

\section{Appendix A.}

This appendix explains how to predict the parameter $\varepsilon$ introduced in Equation (8) and shown in Figure 9. For this purpose we focus on the two cases at $T=85^{\circ} \mathrm{C}$ and to simplify the fit, here we report $\varepsilon$ vs. $\Delta \mu$ :

(i) $C_{0}=9 \%$ behaves like the two cases at $200^{\circ} \mathrm{C}$, i.e. it is almost linear or linear in the case $T=200^{\circ} \mathrm{C}$ and $C_{0}=10 \%$, which can be considered as an asymptotic case, whose prediction is straightforward.

(ii) $C_{0}=4.75 \%$ is a case close to another asymptotic situation which can be simply defined by $\varepsilon=0$. This case is rather delicate to predict with accuracy if available information is not sufficient.

As these two cases are rather different, they require appropriate trial functions to fit the non-linear 
part of their respective curves, but both start with a linear domain. Thus the global fitting procedure remains similar in both cases. The end of the linear part corresponds roughly to the end of the nucleation stage.

- case (i)

If the available information is not sufficient to clearly identify the change of slope of the curve, the half-height of the curve is a good guess. For the non-linear part of the curve, a simple quadratic function satisfying $\varepsilon(0)=0$ is sufficient:

$$
\varepsilon=a \Delta \mu+b \Delta \mu^{2}
$$

From the fit of the linear part we know both the value of $\varepsilon$ and its derivative $\dot{\varepsilon}$ at the point chosen for the transition between the two domains (denoted $\varepsilon^{*}, \dot{\varepsilon}^{*}$ and $\Delta \mu^{*}$, respectively), thus $a$ and $b$ are readily identified:

$$
a=\left(2 \varepsilon^{*}-\dot{\varepsilon}^{*} \Delta \mu^{*}\right) / \Delta \mu^{*}
$$

and

$$
b=\left(\dot{\varepsilon}^{*} \Delta \mu^{*}-\varepsilon^{*}\right) / \Delta \mu^{* 2}
$$

With this type of curve, the risk of error is very low, even if $\Delta \mu^{*}$ is not known with a high accuracy.

- case(ii)

As in the previous case, if $\Delta \mu^{*}$ is not clearly identified, the value corresponding to the half height of the curve is again a good trial value. For the non-linear domain, we choose another simple two-parameters function:

$$
\varepsilon=a \Delta \mu /(\Delta \mu-b)
$$

As previously, $a$ and $b$ are easily identified:

$$
a=-\varepsilon^{* 2} /\left(\dot{\varepsilon}^{*} \Delta \mu^{*}-\varepsilon^{*}\right)
$$

and

$$
b=\dot{\varepsilon}^{*} \Delta \mu^{* 2} /\left(\dot{\varepsilon}^{*} \Delta \mu^{*}-\varepsilon^{*}\right)
$$

Note that this fit is almost correct even at the beginning of the linear domain. This property helps to optimise the choice of $\Delta \mu^{*}$ in case available information is not sufficient.

In Figure A.1, the onset of QE corresponds to the first point (upper right) for $C_{0}=4.75 \%$ : as already mentioned, the information taken before the onset of QE should be disregarded, this is the default value. Nevertheless, for $C_{0}=9 \%$, the onset of $\mathrm{QE}$ corresponds to the second point while the first point is consistent with the rest of the curve: this property will be confirmed for the predictive fit of $M_{1}$ (work in progress). This shows that at very high supersaturation, the default rule does not strictly apply, which is of course an advantage to be taken, but with caution.

In Figure A.1, the arrows indicate the end of the known range of data obtained with small box simulations. As can be seen, these ranges include the transitions between the linear and the non-linear parts of the curves, which shows that the proposed method (section 3.4.2) using one large box and one small box is efficient to provide enough information to ensure a correct choice of $\Delta \mu^{*}$. 


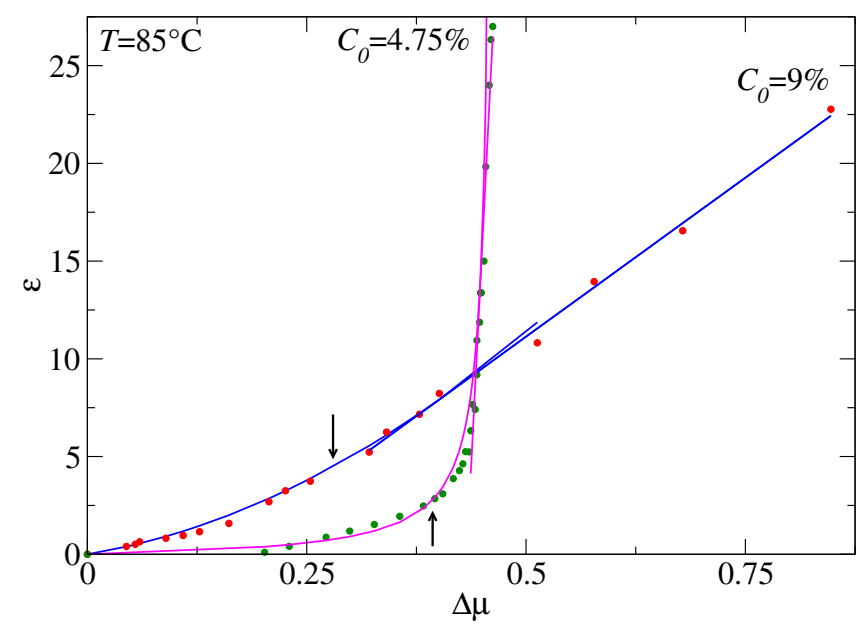

Figure A.1.: Predicting $\varepsilon$ beyond the range of $\Delta \mu$ explored by AKMC simulations. Only the linear domain is assumed to be known (same data than in Figure 9). The half-height of the curves is a good guess for the transition between the linear and the non-linear domains. The arrows indicate the value of $\Delta \mu$ reached by small box simulations when large box simulations are stopped around the onset of $\mathrm{QE}$ (first and second points, starting from the right, for $C_{0}=4.75 \%$ and $C_{0}=9 \%$, respectively). 